\title{
Phase transitions in the Potts spin-glass model
}

\author{
Giancarlo Franzese ${ }^{1}$ and Antonio Coniglio ${ }^{1,2}$ \\ ${ }^{1}$ Dipartimento di Scienze Fisiche, Università di Napoli, Mostra d'Oltremare Padiglione 19 I-80125 Napoli, Italy \\ and Istituto Nazionale di Fisica della Materia, Unità di Napoli, I-80125 Naples, Italy \\ ${ }^{2}$ Istituto Nazionale di Fisica Nucleare, Sezione di Napoli, I-80125 Naples, Italy
}

(Received 3 April 1998)

\begin{abstract}
We have studied the Potts spin glass with two-state Ising spins and $s$-state Potts variables using a cluster Monte Carlo dynamics. The model recovers the $\pm J$ Ising spin glass (SG) for $s=1$ and exhibits for all $s$ a SG transition at $T_{S G}(s)$ and a percolation transition at higher temperature $T_{p}(s)$. We have shown that for all values of $s \neq 1$ at $T_{p}(s)$ there is a thermodynamic transition in the universality class of a ferromagnetic $s$-state Potts model. The efficiency of the cluster dynamics is compared with that of standard spin-flip dynamics.
\end{abstract} [S1063-651X(98)03708-8]

PACS number(s): 64.60.Ak, 75.10.Nr, 02.70.Lq

\section{INTRODUCTION}

In nature there are many examples of glassy systems, i.e., complex systems that exhibit a very slow dynamics that prevents them from reaching the equilibrium state. In this class there is a large variety of systems, such as real glasses, spin glasses, supercooled liquids, polymers, granular material, colloids, ionic conductors, orientational glasses, and vortex glasses [1]. A common feature of these systems is the frustration, which is a competition due to geometry or energy constrains.

Experiments on glasses [2] show that, in a temperaturedriven transition, precursor phenomena start at a temperature well above the ideal glass transition. This temperature depends on experimental conditions and is the onset of some dynamical anomalies, such as nonexponential relaxation functions, anomalous diffusion, and cooling-rate-dependent density. Analogous phenomena are present in spin glasses (SGs) [3], which are a generalization of the Ising model where randomly distributed ferromagnetic and antiferromagnetic interactions give rise to frustration. In particular, experiments [4] and numerical simulations [5] have shown nonexponential autocorrelation functions below a temperature $T^{*}$ well above the transition temperature $T_{S G}$, while above $T^{*}$ only exponential relaxation functions are seen.

To explain this phenomenon, Randeria et al. [6] have suggested that in the SG the nonexponential regime starts at the Griffiths temperature $T_{c}$, i.e., the critical temperature of the ferromagnetic model, due to the presence of randomly large unfrustrated regions, allowed by the disorder in the SG interactions. Campbell and Bernardi [7] have proposed an alternative hypothesis in which the onset $T^{*}$ of nonexponential behavior coincides with the percolation temperature $T_{p}$ of Fortuin-Kasteleyn-Coniglio-Klein [8,9] (FK-CK) clusters (defined below). The idea of Ref. [7] is that in the SG the accessible phase space is simply connected above $T_{p}$, while it is not below $T_{p}$. Therefore, for $T<T_{p}$ a local Monte Carlo (MC) dynamics performs a random walk on a ramified percolatinglike structure with many time scales, giving rise to nonexponential relaxation functions.

Since $T_{p}$ is less than but close to $T_{c}$ and $T^{*}$ is difficult to localize, it is not possible to exclude the $T^{*}=T_{c}$ hypothesis. However, numerical results $[10,11]$ on fully frustrated models, without disorder, where the Randeria et al. argument does not apply, are consistent with the $T^{*}=T_{p}$ scenario. In Refs. $[10,11]$ this result is given for both spin-flip and bondflip dynamics. The latter is strictly related to the bond frustrated percolation (FP) where frustrated loops, i.e., closed paths of connected bonds covering an odd number of negative interactions, have zero weight [10]. The FP model and the $\pm J$ SG model can be recovered as particular cases of a $2 s$-state Potts spin glass (PSG) [12], respectively, for $s$ $=1 / 2$ and $s=1$.

In this paper we will show numerical results on the static properties of the PSG model in two dimensions (2D). For any $s$ the model exhibits a $\mathrm{SG}$ transition at a temperature $T_{S G}(s)$ (in 2D $T_{S G}=0$ for any $s$ ) and a FK-CK percolation transition at higher temperature $T_{p}(s)$. For $s \neq 1$ the higher transition corresponds to a real thermodynamic transition of an $s$-state Potts model [13]. To make the algorithm faster we use the Swendsen-Wang (SW) [14] cluster MC dynamics that prevent the slowing down for temperatures near $T_{p}(s)$. Dynamical properties of the model for $s=2$ are given in Ref. [15], where we have shown that autocorrelation times diverge at $T_{p}$ and, as in SG model, nonexponential relaxation functions are present below $T_{p}$. These results on the SG model [7], the FP model [10], fully frustrated systems [11], and the PSG model [15] suggest that the FK-CK percolation may play a role in the context of precursor phenomena since below $T_{p}$ the frustration is present at all length scales by means of the FK-CK percolating cluster, which cannot include frustrated loops.

In Sec. II we define the Hamiltonian and review some theoretical results. In Sec. III we present the numerical results for the model in finite dimensions. In Sec. IV we define the SW dynamics and compare it with the spin-flip dynamics, verifying the efficiency of the cluster dynamics. In Sec. $\mathrm{V}$ we give the conclusions. 


\section{HAMILTONIAN FORMALISM}

The PSG model is defined by the Hamiltonian

$$
H=-s J \sum_{\langle i, j\rangle}\left[\delta_{\sigma_{i} \sigma_{j}}\left(\epsilon_{i, j} S_{i} S_{j}+1\right)-2\right],
$$

where associated with each lattice site is an Ising spin $S_{i}$ $= \pm 1$ and an $s$-state Potts spin $\sigma_{i}=1, \ldots, s$. The sum is extended over all nearest-neighbor (NN) sites, $\epsilon_{i, j}= \pm 1$ is a random quenched variable, and $J$ is the strength of interaction. The model is a superposition of a ferromagnetic $s$-state Potts model [13] and a $\pm J$ Ising SG [3] and for $\delta_{\sigma_{i} \sigma_{j}}=1$ (i.e., $s=1$ ) recovers the $\pm J$ Ising SG Hamiltonian.

Following Ref. [12], it is possible to define FK-CK clusters on this model, activating a bond, between NN sites with both the SG interaction and Potts interaction satisfied, with probability

$$
p=1-e^{-2 s J / k_{B} T}
$$

and defining a cluster as the maximal set of connected bonds.

For a given set of interactions $\left\{\epsilon_{i, j}\right\}$ it is possible to shown [12] that $Z$ can be expressed in terms of bond configurations $C$,

$$
Z\left\{\epsilon_{i, j}\right\}=\sum_{\left\{S_{i}, \sigma_{i}\right\}} e^{-H / k_{B} T}=\sum_{C} W_{s}(C),
$$

where $W_{s}(C)=0$ if $C$ includes any frustrated loop; otherwise

$$
W_{s}(C)=p^{|C|}(1-p)^{|A|}(2 s)^{N(C)},
$$

where $p$ is given in Eq. (2), $N(C)$ is the number of clusters in the configuration $C,|C|$ is the number of bonds, and $|C|+|A|$ is the total number of interactions. Let us observe that, while the Hamiltonian (1) is defined only for integer values of $s$, Eq. (3) is meaningful for every value of $s$ and for $s=1 / 2 \mathrm{Eq}$. (3) gives the partition function of bond FP where a bond configuration without frustrated loops has a weight $W(C)=e^{\beta \mu|C|}$ with $\beta \mu=\ln \left(e^{\beta J}-1\right)$, while a bond configuration with frustrated loops has a zero weight. Furthermore, in the limit $s \rightarrow 0 \mathrm{Eq}$. (3) gives the partition function of the tree percolation [13], where any bond configuration with a loop is excluded.

An exact renormalization group ( $\mathrm{RG}$ ) analysis on a hierarchical lattice [16] has predicted for the PSG two critical temperatures. The lower temperature $T_{S G}(s)$ corresponds to a $S G$ transition in the universality class of the $\pm J$ Ising $S G$ and the higher $T_{p}(s)$ to a percolation transition in the universality class of a ferromagnetic $s$-state Potts model. The same results are given for the fully frustrated version of the model studied with a mean field approach [17].

Looking at the partition function (3), one should expect a singularity at $T_{p}(s)$, for any $s \neq 0$, due to the singularity in the number of clusters $N(C)$. Nevertheless, this singularity has never been observed in the case of SG $(s=1)$. In fact, the RG calculations in the case $s=1$ show a singularity at $T_{S G}$ for the SG free energy and no singularity at $T_{p}(1)$. This result is interpreted in Ref. [16] supposing that the free energy of the Hamiltonian (1) has the form

$$
F_{s}(T)-F_{s}\left(T_{p}\right) \sim A(s)\left[T-T_{p}(s)\right]^{2-\alpha(s)},
$$

where $A(s)$ is an amplitude that vanishes for $s \rightarrow 1$ and $\alpha(s)$ is the specific heat exponent.

\section{MONTE CARLO RESULTS}

We have done our simulation using the SW Monte Carlo cluster dynamics [14] described in Sec. IV. As we will show, the SW dynamics is faster than standard local dynamics, such as spin-flip dynamics, but suffers a slowing down near the SG critical temperature. Nevertheless, since the SG transition in 2D occurs at $T_{S G}=0$ [18] and we are interested in studying the system near the percolation transition at $T_{p}$ $>T_{S G}$, the SW dynamics is particularly indicated.

We have performed numerical simulation of the PSG model for $s=2,7$, and 50 on a two-dimensional square lattice with linear sizes ranging from $L=10$ to 60 lattice steps and with quenched random interaction configurations $\left\{\epsilon_{i j}\right\}$. Defining as a MC step an update of all the spins of the system, we have discarded the data of the first $7500 \mathrm{MC}$ steps and have collected data over 15000,25000 , or 50000 MC steps, depending on the temperatures and sizes.

For each $s$ we have calculated the Binder parameter for the energy density $E[19]$ defined as

$$
V=1-\frac{\left\langle E^{4}\right\rangle}{3\left\langle E^{2}\right\rangle^{2}},
$$

where the symbol angular brackets stand for the thermal average. This quantity allows us to localize the transition and to distinguish between first-order and second-order phase transition. In fact, for a second-order phase transition in the limit $L \rightarrow \infty$ it is $V=2 / 3$ for all temperatures, while for a first-order phase transition it is

$$
\frac{2}{3}-V^{\min }=\frac{1}{3} \frac{\left(E_{+}-E_{-}\right)^{2}\left(E_{+}+E_{-}\right)^{2}}{\left(E_{+}^{2}+E_{-}^{2}\right)^{2}}
$$

where $V^{\text {min }}$ is the minimum value of $V$ (occurring at the phase transition temperature) and $E_{+}-E_{-}$is the energy jump, related to the latent heat, at the same temperature.

To estimate the thermodynamic critical exponents for the second-order phase transition we have measured the Potts order parameter

$$
M=\frac{s \max _{i}\left(M_{i}\right)-1}{s-1}
$$

(where $i=1, \ldots s$ and $M_{i}$ is the density of Potts spins in the $i$ th state), the susceptibility

$$
\chi=\left[\frac{\left\langle M^{2}\right\rangle-\langle M\rangle^{2}}{N}\right]^{1 / 2}
$$

(where $N$ is the total number of Potts spins), and the specific heat

$$
C_{H}=\left[\frac{\left\langle E^{2}\right\rangle-\langle E\rangle^{2}}{N}\right]^{1 / 2} .
$$




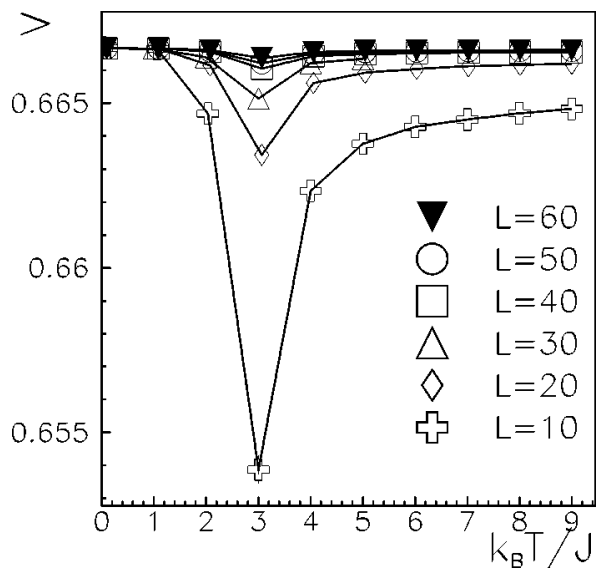

FIG. 1. PSG model for $s=2$ : Binder parameter $V$ vs dimensionless temperature $k_{B} T / J$ for $L=10-60$. Errors are smaller than the symbol sizes. Lines are only guides for the eyes.

Furthermore, to estimate the percolation critical exponents we have calculated the percolation probability per spin

$$
P=1-\sum_{k} k n_{k}
$$

(where $k$ is the cluster size and $n_{k}$ is the density of clusters of size $k$ ), the mean cluster size

$$
S=\sum_{k} k^{2} n_{k}
$$

and the number of clusters

$$
N_{c}=\sum_{k} n_{k}
$$

\section{A. Results for $s=2$}

In Fig. 1 we show the Binder parameter for the case $s$ $=2$ for system sizes $L=10-60$. It is possible to see that $V$ for small sizes has a minimum at $k_{B} T / J \simeq 3.0$ and that for greater sizes it becomes constant for all temperatures, revealing a second-order phase transition. Therefore, we can make a standard scaling analysis [20] for the thermodynamic quantities.

In particular, by definition of critical exponents $\nu$ it is

$$
\xi \sim\left|T-T_{s}\right|^{-\nu}
$$

where $\xi$ is the correlation length and $T_{s}=\lim _{L \rightarrow \infty} T_{s}(L)$ with $T_{s}(L)$ the finite size critical temperature of the PSG model. Analogously to the definitions of the other critical exponents $\beta, \gamma$, and $\alpha$, we get

$$
\begin{gathered}
M \sim\left|T-T_{s}\right|^{\beta} \sim \xi^{-\beta / \nu}, \\
\chi \sim\left|T-T_{s}\right|^{-\gamma} \sim \xi^{\gamma / \nu}, \\
C_{H} \sim\left|T-T_{s}\right|^{-\alpha} \sim \xi^{\alpha / \nu} .
\end{gathered}
$$

From the standard scaling analysis applied to finite systems [20] we expect for $M$

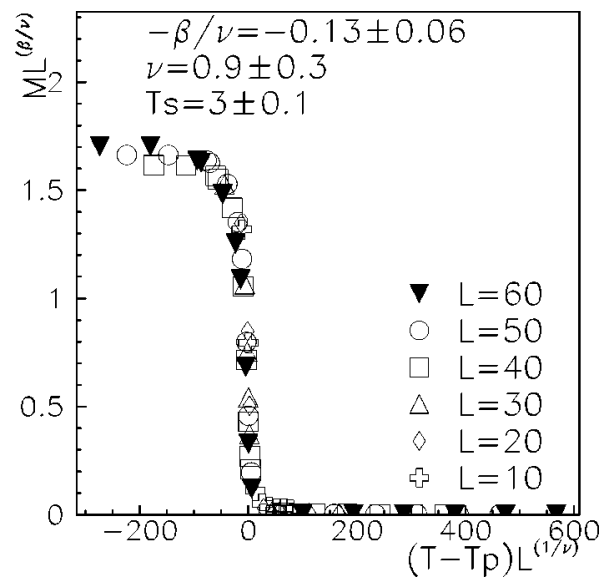

FIG. 2. PSG model with $s=2$ : Data collapse for $M$ for systems sizes $L=10-60$. Temperatures are in $J / k_{B}$ units. The scaling parameters are given in the figure.

$$
M \sim L^{-\beta / \nu} f_{M}\left(\left(T-T_{s}\right) L^{1 / \nu}\right),
$$

where $f_{M}(x)$ is an universal function of the dimensionless variable $x$. Analogous scaling functions are expected for the other thermodynamic quantities. Tuning the values of critical exponents and $T_{s}$, it is possible to verify the scaling hypothesis, as Eq. (18), from the MC data. The values for which the data collapse give the estimates of the critical exponents and of $T_{s}$.

In Figs. 2-4 we show the data collapses for system sizes $L=10-60$. The estimated scaling parameters [21] are given in Table I.

The estimated critical exponents are compatible, within the errors, with the expected values for a Potts model with the $s=2$ state, i.e., an Ising model, in 2D: $\alpha=0, \beta=1 / 8$ $=0.125, \gamma=7 / 4=1.75$, and $\nu=1$ [22]. The estimated critical temperature is $k_{B} T_{s} / J=2.95 \pm 0.15$.

For the FK-CK percolation quantities we have described the critical behavior of $P$ and $S$ introducing a percolation set of critical exponents $\left(\alpha_{p}, \beta_{p}, \gamma_{p}\right.$, and $\left.\nu_{p}\right)$ defined by the relation

$$
\xi_{p} \sim\left|T-T_{p}\right|^{-\nu_{p}}
$$

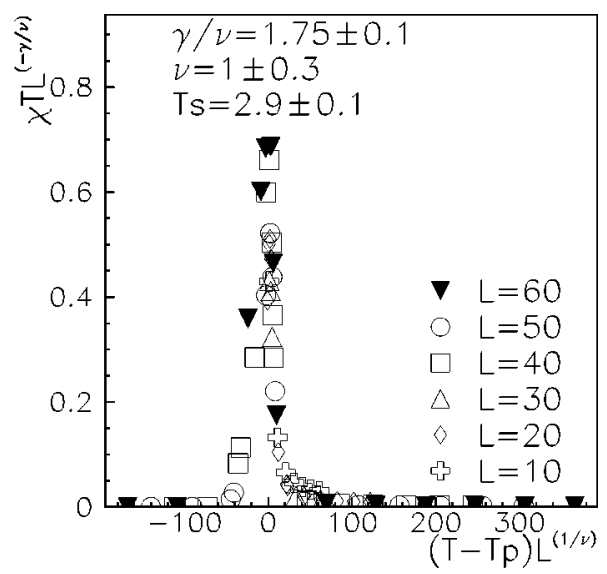

FIG. 3. PSG model with $s=2$ : Data collapse for $\chi$ for systems sizes $L=10-60$. Temperatures are in $J / k_{B}$ units. The scaling parameters are given in the figure. 


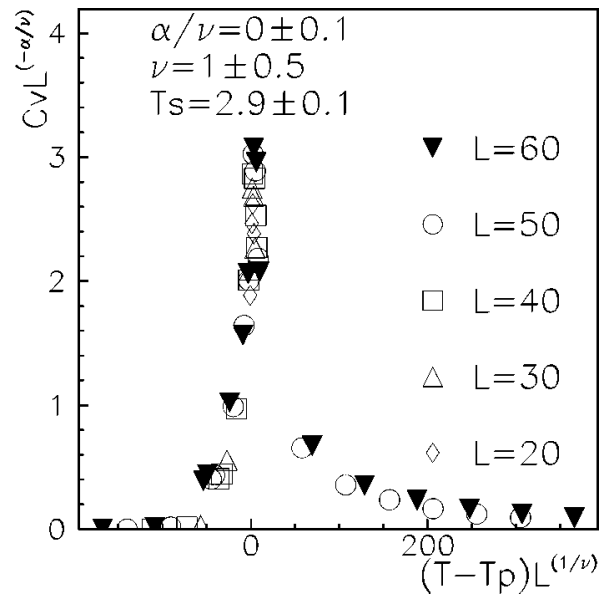

FIG. 4. PSG model with $s=2$ : Data collapse for $C_{H}$ for systems sizes $L=10-60$. Temperatures are in $J / k_{B}$ units. The scaling parameters are given in the figure.

where $\xi_{p}$ is the connectedness length of the clusters and $T_{p}$ $=\lim _{L \rightarrow \infty} T_{p}(L)$, with $T_{p}(L)$ the finite size percolation temperature,

$$
\begin{aligned}
& P \sim\left|T-T_{p}\right|^{\beta_{p} \sim \xi_{p}^{-\beta_{p} / \nu_{p}},} \\
& S \sim\left|T-T_{p}\right|^{-\gamma_{p} \sim \xi_{p}^{\gamma_{p}} / \nu_{p}} .
\end{aligned}
$$

A standard scaling analysis for finite systems [23] is applied also in this case and the results are summarized in Figs. 5 and 6. The estimated scaling parameters are given in Table II. All the estimated exponents are compatible, within the errors, with the corresponding thermodynamic parameters for the two-dimensional Ising model and the numerical estimate for the percolation temperature is $k_{B} T_{p} / J=2.925$ \pm 0.075 , consistent with the estimates of $T_{s}$.

\section{B. Results for $s=7$ and $\mathbf{5 0}$}

In Fig. 7 we show the Binder parameter $V$ for the PSG model with $s=7$ and 50 for system sizes $L=10-50$. The fact that $V$ has a nonvanishing minimum for every size reveals that there is a first-order phase transition. In this case there is no diverging length; therefore, the scaling analysis cannot be applied. This kind of transition is characterized by the finite size relations [20]

$$
C_{H}\left(T_{s}(L), L\right) \simeq \max _{T}\left[C_{H}(T, L)\right] \sim L^{D}
$$

(where $D$ is the Euclidean dimension) for the maximum of finite size $C_{H}(L)$ (see Table III) and the relation

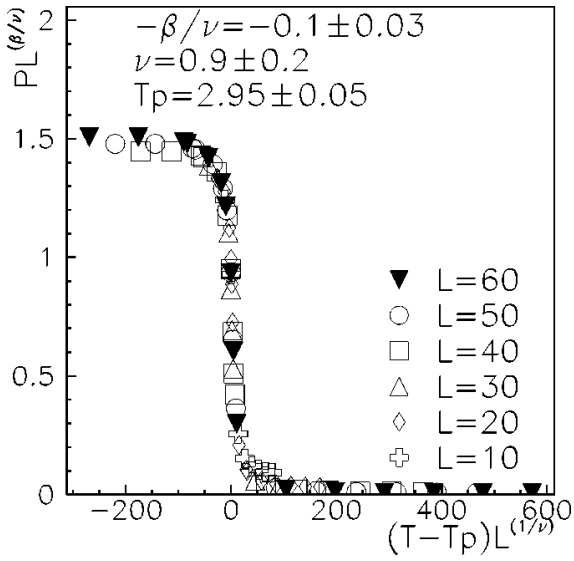

FIG. 5. PSG model with $s=2$ : Data collapses for $P$ for systems sizes $L=10-60$. Temperatures are in $J / k_{B}$ units. The scaling parameters are given in the figure. The indices $p$ are omitted for the critical exponents.

$$
T_{\max }(L)-T_{\max }(\infty) \sim L^{-D}
$$

where $T_{\max }(L)$ is the temperature of the maximum of $C_{H}$ (or of the mean cluster size $S$ ) for the size $L$ and $T_{\max }(\infty)$ is the corresponding value in the thermodynamic limit, i.e., the corresponding transition temperature $T_{s}(s)$ [or $\left.T_{p}(s)\right]$. Therefore, $T_{s}(s)$ and $T_{p}(s)$ can be evaluated by linear fits with one free parameter. The data are given in Table IV and the results are for $s=7, T_{s}=T_{p}=7.5 \pm 0.1$ and $s=50, T_{s}$ $=T_{p}=35.0 \pm 0.1$.

The results are summarized in the phase diagram in Fig. 8: For every $s$ the high-temperature phase is disordered and nonpercolating; decreasing the temperature, there is a second- or first-order phase transition (depending on $s$ ) at $T_{p}$ corresponding to the percolation of FK-CK clusters and to the ordering of Potts variables; at lower temperature there is the SG transition (which in 2D occurs at $T=0$ [18]). For a fixed realization of $\left\{\epsilon_{i j}\right\}$ it is possible to show [24] that each critical point is characterized by a diverging critical length. At $T_{p}(s)$ the linear size of FK-CK clusters associated with the pair connectedness function diverges, while at $T_{S G}(s)$ the linear size of correlated regions diverges.

It is interesting to note that the behavior of $T_{p}(s)$ can be obtained from the exact expression of the transition temperature of a ferromagnetic $2 s$-state Potts model [13] only by renormalizing the number of states, i.e.,

$$
\frac{T_{p}}{2 s a}=\frac{1}{\ln (1+\sqrt{2 s a})},
$$

with $a=0.803 \pm 0.003$ (choosing $J=k_{B}$ ).

TABLE I. Estimated critical exponents and critical temperatures $T_{s}$ for thermodynamic quantities $M, \chi$, and $C_{H}$ for the PSG model with $s=2$.

\begin{tabular}{cccccc}
\hline \hline $\begin{array}{c}\text { Thermodynamic } \\
\text { quantity }\end{array}$ & $\nu$ & $\alpha / \nu$ & $\beta / \nu$ & $\gamma / \nu$ & $k_{B} T_{s} / J$ \\
\hline$M$ & $0.9 \pm 0.3$ & & $0.13 \pm 0.06$ & & $3.0 \pm 0.1$ \\
$\chi$ & $1.0 \pm 0.3$ & & & $1.75 \pm 0.10$ & $2.9 \pm 0.1$ \\
$C_{H}$ & $1.0 \pm 0.5$ & $0.0 \pm 0.1$ & & & $2.9 \pm 0.1$ \\
\hline \hline
\end{tabular}




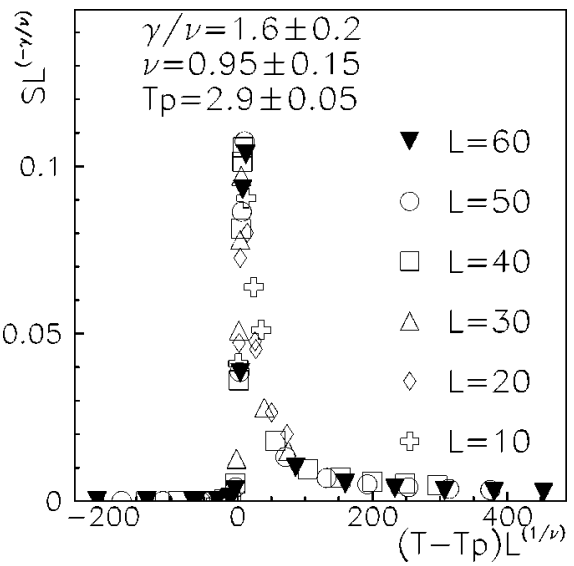

FIG. 6. PSG model with $s=2$ : Data collapses for $S$ for systems sizes $L=10-60$. Temperatures are in $J / k_{B}$ units. The scaling parameters are given in the figure. The indices $p$ are omitted for the critical exponents.

\section{COMPARISON WITH SPIN-FLIP DYNAMICS}

The MC dynamics used to study the equilibrium properties of the PSG model is the SW cluster MC dynamics. The $\mathrm{SW}$ dynamics is performed in two steps. The first step is to construct the FK-CK cluster configuration $C$, given an Ising and a Potts spin configuration $\left\{S_{i}, \sigma_{i}\right\}$, activating bonds with the probability in Eq. (2) between NN sites when both Ising and Potts spins satisfy the interaction and with zero probability otherwise. The second step consists in reversing all the spins in a cluster at the same time with probability $1 / 2$ for each cluster. The sequence of the first and the second steps applied to the whole system constitutes a MC step, which is the chosen unit of time.

This dynamics completely overcomes the problem of critical slowing down for the unfrustrated spin models [14], while it suffers from a diverging correlation time if applied to frustrated systems near a critical point. This inefficiency is a consequence of the fact that the FK-CK clusters used in the SW dynamics no longer represent, in frustrated models, the regions of correlated spins near a critical point [12] and their percolation temperature $T_{p}$ is greater than the critical temperature. In particular, this is true for the SG model for which an efficient cluster MC dynamics does not yet exist except for 2D [26], while efficient cluster dynamics have been proposed for systems with frustration but without disorder [27,28]. Nevertheless, in SGs for temperatures well above the critical temperature $T_{S G}$ and near $T_{p}$ the SW dynamics is still efficient, consistently with the general observation that the cluster dynamics is efficient at least for temperatures above the percolation temperature [28].

On the other hand, in Ref. [15] we have shown that the local spin-flip (SF) MC dynamics [20] in the case of the PSG

TABLE II. Estimated percolation exponents and percolation temperature $T_{p}$ for $P$ and $S$ for the PSG model with $s=2$.

\begin{tabular}{ccccc}
\hline \hline $\begin{array}{c}\text { Percolation } \\
\text { quantity }\end{array}$ & $\nu_{p}$ & $\beta_{p} / \nu_{p}$ & $\gamma_{p} / \nu_{p}$ & $k_{B} T_{p} / J$ \\
\hline$P$ & $0.9 \pm 0.2$ & $0.10 \pm 0.03$ & & $2.95 \pm 0.05$ \\
$S$ & $0.95 \pm 0.15$ & & $1.6 \pm 0.2$ & $2.90 \pm 0.05$ \\
\hline \hline
\end{tabular}
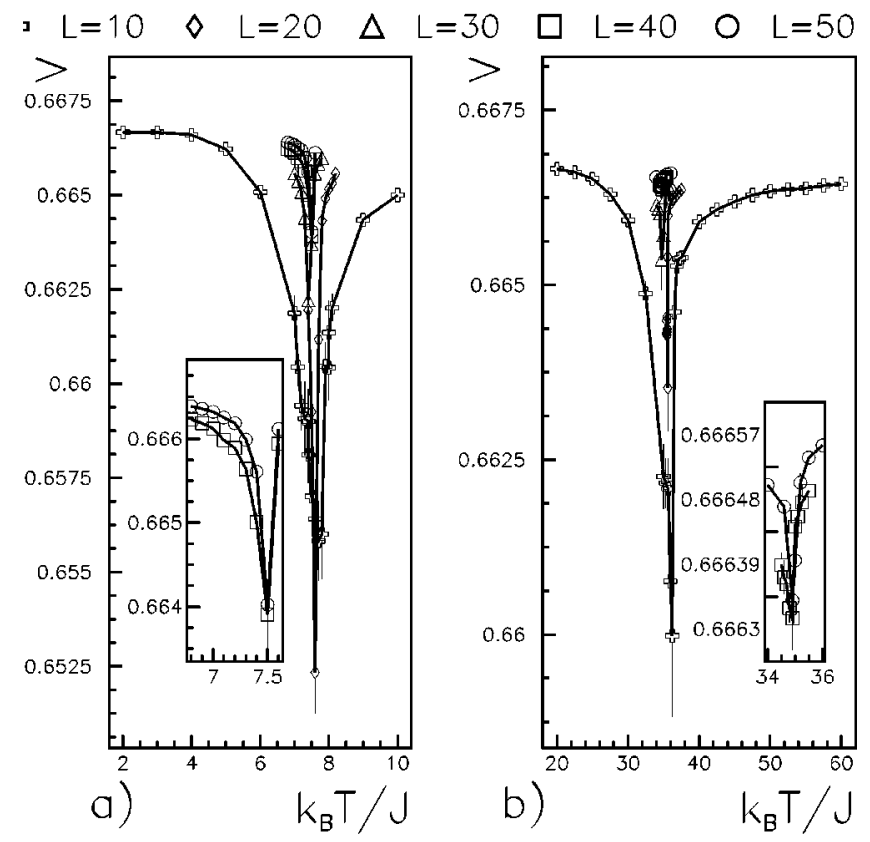

FIG. 7. PSG model: Binder parameter $V$ vs dimensionless temperature $k_{B} T / J$ for (a) $s=7$ and (b) $s=50$, for $L=10-50$. The insets show the particulars for $L=40$ and 50. Where not shown, the errors are smaller than the symbol sizes. Lines are only guides for the eyes.

model with $s=2$ is characterized near $T_{p}$ by diverging correlation times. To compare the efficiency of SW dynamics to that of SF dynamics we have studied for the case $s=2$ the correlation functions at the equilibrium, which for a generic observable $A$ is defined as

$$
f_{A}(t)=\left[\frac{\left\langle\delta\left(t+t_{0}\right) \delta\left(t_{0}\right)\right\rangle}{\left\langle\delta\left(t_{0}\right)^{2}\right\rangle}\right],
$$

where $\delta(t)=A(t)-\langle A\rangle$ and $t_{0}$ is the equilibration time. As observables we have choose the Potts order parameter $M$ and the energy $E$ of the whole system.

We have also studied the time-dependent nonlinear susceptibility for a quenched interaction configuration

$$
\chi_{S G}(t)=\frac{1}{N}\left\langle\left[\sum_{i} S_{i}\left(t+t_{0}\right) S_{i}\left(t_{0}\right)\right]^{2}\right\rangle,
$$

where $N$ is the total number of spins. The normalized correlation function is

$$
f_{\chi}=\frac{\chi_{S G}(t)-\chi_{S G}(t=\infty)}{\chi_{S G}(0)-\chi_{S G}(t=\infty)},
$$

with $\chi_{S G}(0)=N$.

TABLE III. Maxima of $C_{H}$ for $s=7$ and $s=50$ for $L=30,40$, and 50 .

\begin{tabular}{lccc}
\hline \multicolumn{1}{c}{$L$} & 30 & 40 & 50 \\
\hline $\max _{T} C_{H}(s=7) / L^{2}$ & $27 \pm 3$ & $26 \pm 3$ & $33 \pm 3$ \\
$\max _{T} C_{H}(s=50) / L^{2}$ & $7.4 \pm 0.6$ & $8.4 \pm 0.9$ & $7.3 \pm 0.7$ \\
\hline \hline
\end{tabular}


TABLE IV. Temperatures (in $J / k_{B}$ units) of maxima of $C_{H}$ and $S$ for $s=7$ and 50 for $L=10,20,30,40$, and 50 .

\begin{tabular}{lccccc}
\hline \hline \multicolumn{1}{c}{$L$} & 10 & 20 & 30 & 40 & 50 \\
\hline$T_{\max } C_{H}(s=7)$ & 7.8 & 7.6 & 7.5 & 7.5 & 7.5 \\
$T_{\max S}(s=7)$ & 7.9 & 7.7 & 7.5 & 7.5 & 7.52 \\
$T_{\max } C_{H}(s=50)$ & 36.56 & 35.57 & 34.92 & 34.90 & 35.00 \\
$T_{\max S}(s=50)$ & 36.56 & 35.60 & 35.00 & 35.00 & 35.00 \\
\hline \hline
\end{tabular}

For both the SW and the SF dynamics we have measured the integral correlation time defined as

$$
\tau_{\text {int }}=\lim _{t_{\max } \rightarrow \infty} \frac{1}{2}+\sum_{t=0}^{t_{\max }} f(t)
$$

where $f$ is the generic correlation function. We have considered systems with lattice sizes $L \leqslant 30$ at temperatures above and below $T_{p}$. The data for the SF dynamics are averaged over 32 different quenched interaction configurations since the local updating of this dynamics strongly depends on the local fluctuation of the frustration. On the other hand, the results on global SW dynamics turns out to be "robust" with respect to the interaction configuration average, in the sense that the fluctuations of $\tau_{i n t}$ are within the errors estimated on the basis of a single interaction configuration analysis.

The simulations have been done with an annealing method, i.e., with a slow cooling of the system at each temperature. For the SW cluster dynamics $5 \times 10^{3} \mathrm{MC}$ steps turn out to be enough to equilibrate the system at the considered temperatures and the averages are done using the the data for the following $5 \times 10^{4} \mathrm{MC}$ steps. For the SF dynamics we have discarded the first $10^{4} \mathrm{MC}$ steps (defined as the local update of any spin in the system) and recorded the data for $5 \times 10^{5}$ MC steps.

In Table $\mathrm{V}$ we show the results for $L=30$. Analogous results have been found for smaller systems.

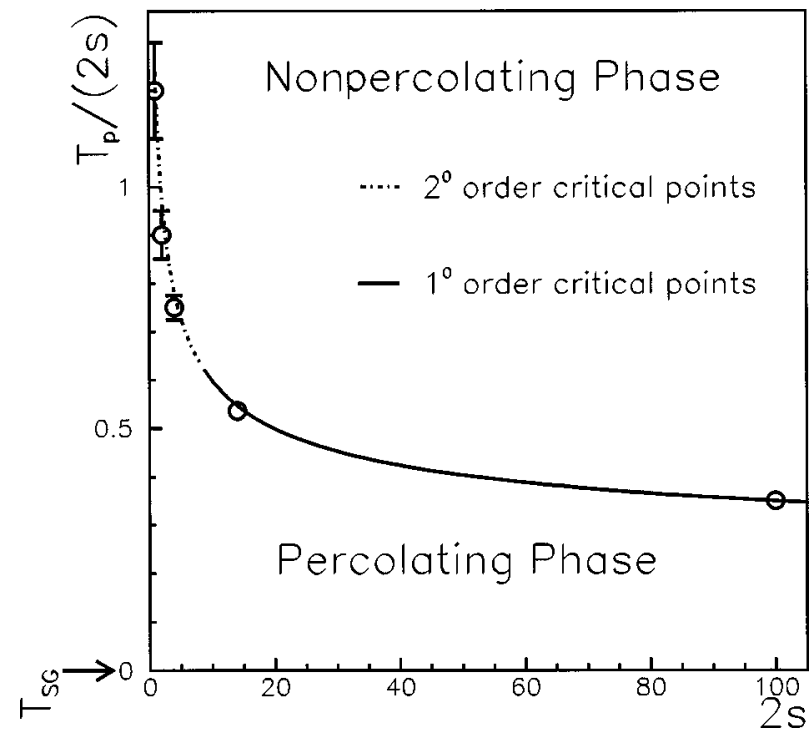

FIG. 8. PSG model: Numerical phase diagram in 2D. The data are fitted with $T_{p} / 2 s=a /[\ln (1+\sqrt{2 s a})]$ with $a=0.803 \pm 0.003$ (choosing $J=k_{B}$ ). Data for $s=1 / 2$ and $s=1$ are from Ref. [25]. Where not shown, the errors are smaller than the symbol sizes.
TABLE V. Integral correlation times for a PSG model with $s$ $=2$ and $L=30$ for the Swendsen-Wang (SW) cluster MC dynamics and local spin-flip (SF) MC dynamics for temperatures above and below $T_{p}(L=30) \simeq 2.95 \mathrm{~J} / k_{B}$.

\begin{tabular}{lccc}
\hline \hline \multicolumn{1}{c}{$k_{B} T / J$} & 2.75 & 3.00 & 3.25 \\
\hline$\tau_{M}(\mathrm{SW})$ & $3.08 \pm 0.02$ & $3.65 \pm 0.03$ & $1.81 \pm 0.01$ \\
$\tau_{M}(\mathrm{SF})$ & $76.8 \pm 0.3$ & $575.8 \pm 0.6$ & $180.3 \pm 0.2$ \\
$\tau_{E}(\mathrm{SW})$ & $9.32 \pm 0.02$ & $8.53 \pm 0.07$ & $4.45 \pm 0.04$ \\
$\tau_{E}(\mathrm{SF})$ & $19.0 \pm 0.1$ & $67.5 \pm 0.1$ & $20.9 \pm 0.5$ \\
$\tau_{\chi}(\mathrm{SW})$ & $9.93 \pm 0.07$ & $2.423 \pm 0.002$ & $1.585 \pm 0.001$ \\
$\tau_{\chi}(\mathrm{SF})$ & $16.89 \pm 0.02$ & $7.06 \pm 0.03$ & $3.97 \pm 0.01$ \\
\hline \hline
\end{tabular}

The data show that, while the SF correlation times for $M$ and $E$ grow abruptly near $T_{p}$ where both thermodynamic and percolation transitions occur, the SW correlation times only show a slow trend to increase for decreasing temperatures, being smaller than the corresponding SF data by at least an order of magnitude. Even for $\tau_{\chi}$, which for a SF starts to be nonzero below $T_{p}$, the SW dynamics shows smaller correlation times. For temperatures well below $T_{p}$ it is possible to see that the SW dynamics is characterized by long autocorrelation times, like the SF dynamics. Therefore, at least for temperatures not too much below $T_{p}$, the $\mathrm{SW}$ dynamics turns out to be more efficient than local SF dynamics. In particular, near the thermodynamic transition at $T_{p}$ the SW dynamics completely overcomes the critical slowing down problem in the PSG model, as for the unfrustrated models, even if frustration is present via the random interactions of the Ising spins.

\section{CONCLUSIONS}

Precursor phenomena characterize the paramagnetic phase of a spin glass. In particular, experiments and local Monte Carlo simulations show the presence of stretched exponential autocorrelation functions well above the SG transition temperature $T_{S G}$ [7]. The relation of the onset $T^{*}$ of these precursor phenomena to any thermodynamic transition and the localization of $T^{*}$ are still matters of debate $[7,15]$. Much numerical evidence on disordered [7] and deterministic frustrated models $[10,11]$ have shown that $T^{*}$ is consistent with the percolation temperature $T_{p}$ of the FortuinKasteleyn-Coniglio-Klein clusters. In particular, a generalization of the $\pm J$ Ising SG to a $2 s$-state Potts SG, which recovers the SG for $s=1$, has shown that for $s=2$, like for $s=1$ [7], the $T^{*}=T_{p}$ hypothesis is numerically verified [15]. In this paper, using very efficient cluster Monte Carlo dynamics, we have shown that in $2 \mathrm{D}$ for any $s \neq 1$ the percolation transition corresponds to a real thermodynamic transition in the universality class of the $s$-state ferromagnetic Potts model. In particular, we have considered the cases $s=2, s=7$ and 50 , where at $T_{p}(s)$ a second-order and, respectively, a first-order phase transition occurs. Exact renormalization group calculations on hierarchical lattices [16] and a mean field analysis for a version of the model without disorder [17] have shown the same scenario.

All these results suggest that the percolation transition may play a role in the precursor phenomena even in the SG case $(s=1)$, where no thermodynamic transition occurs at $T_{p}$. This idea arises from the observation that, even in SGs, 
below $T_{p}$ the frustration starts to be manifested on all length scales by means of the FK-CK clusters, which cannot include frustrated loops. Therefore, the scenario presented is that for $s \neq 1$ at the percolation temperature $T_{p}$ there is a thermodynamic transition with associated dynamical anomalies that "vanishes" for $s=1$ leaving the dynamical behavior unchanged.

\section{ACKNOWLEDGMENTS}

We would like to thank A. De Candia and V. Cataudella for kindly supplying the data for $s=1 / 2$ and $s=1$ in Fig. 8 . This work was partially supported by the TMR network through Contract No. ERBFMRXCT 980183.
[1] See, for example, Proceedings of 14th Citges Conferences on Complex Behavior of Glassy Systems, (Springer-Verlag, Berlin, 1996).

[2] M. D. Ediger, C. A. Angell, and S. R. Nagel, J. Phys. Chem. 100, 13200 (1996); C. A. Angell, Science 267, 1924 (1995); Relaxation in Complex Systems, edited by K. L. Ngai and G. B. Wright (Office of Naval Research, Washington, DC, 1984).

[3] M. Mezard, G. Parisi, and M. A. Virasoro, Spin Glass Theory and Beyond (World Scientific, Singapore, 1987).

[4] F. Mezei and A. P. Murani, J. Magn. Magn. Mater. 14, 211 (1979).

[5] A. T. Ogielski, Phys. Rev. B 32, 7384 (1985).

[6] M. Randeria, J. P. Sethna, and R. G. Palmer, Phys. Rev. Lett. 54, 1321 (1985).

[7] I. A. Campbell and L. Bernardi, Phys. Rev. B 50, 12643 (1994).

[8] C. M. Fortuin and P. W. Kasteleyn, Physica (Amsterdam) 57, 536 (1972).

[9] A. Coniglio and W. Klein, J. Phys. A 13, 2775 (1980).

[10] A. Fierro, A. de Candia, and A. Coniglio, Phys. Rev. E 56, 4990 (1997).

[11] A. Fierro, G. Franzese, A. de Candia, and A. Coniglio, cond-mat/9803202.

[12] A. Coniglio, F. di Liberto, G. Monroy, and F. Peruggi, Phys. Rev. B 44, 12605 (1991).
[13] F. Wu, Rev. Mod. Phys. 54, 235 (1982).

[14] R. H. Swendsen and J. S. Wang, Phys. Rev. Lett. 58, 86 (1987).

[15] G. Franzese and A. Coniglio, cond-mat/9803043.

[16] U. Pezzella and A. Coniglio, Physica A 237, 353 (1997).

[17] F. di Liberto and F. Peruggi, Physica A 248, 273 (1998).

[18] R. N. Bath and A. P. Young, Phys. Rev. B 37, 5606 (1988).

[19] K. Binder, Z. Phys. B 43, 119 (1981).

[20] K. Binder and D. W. Hermann, Monte Carlo Simulation in Statistical Physics (Spinger-Verlag, Berlin, 1988).

[21] The errors are overestimated since their evaluation is done by changing a parameter and taking the other fixed at the best value.

[22] L. Onsager, Phys. Rev. 65, 117 (1944).

[23] A. Aharony and D. Stauffer, Introduction to Percolation Theory (Taylor \& Francis, London, 1994).

[24] V. Cataudella, A. Coniglio, L. de Arcangelis, and F. Peruggi, Physica A 192, 167 (1993).

[25] A. de Candia, V. Cataudella, and A. Coniglio (unpublished).

[26] R. H. Swendsen and J. S. Wang, Phys. Rev. Lett. 57, 2607 (1986); J. S. Wang and R. H. Swendsen, Phys. Rev. B 38, 4840 (1988).

[27] V. Cataudella, G. Franzese, M. Nicodemi, A Scala, and A. Coniglio, Phys. Rev. Lett. 72, 1541 (1994); Phys. Rev. E 54, 175 (1996).

[28] G. Franzese (unpublished). 